\title{
El papel didáctico de las teselaciones para el estudio de los polígonos en el bachillerato
}

\author{
Josefa Osuna Márquez \\ Cristina Villalva y Gutiérrez \\ Universidad del Valle de México \\ Universidad de Sonora \\ e-mail: osunamj@gmail.com
}

\begin{abstract}
Resumen
Esta es la descripción del diseño de una secuencia de actividades didácticas fundamentada en la Teoría Antropológica de lo Didáctico y basada en el estudio de teselaciones poligonales, que busca promover el desarrollo de los procesos cognitivos del pensamiento geométrico que son propuestos y clasificados según Raymond Duval, en procesos de visualización, de construcción y de razonamiento. Se tiene la hipótesis de que al ubicar el desarrollo del pensamiento geométrico - así caracterizado- como objetivo central, se consigue consecuentemente incidir en el desarrollo de competencias que plantea el enfoque impulsado por la Reforma Integral de Educación Media Superior.
\end{abstract}

\section{Introducción}

Pese a su presencia constante en la vida cotidiana, la enseñanza de la geometría vive una crisis, como lo denunciara Vinicio Villani [7] en Perspectivas en la Enseñanza de la Geometría para el Siglo XXI. Dicha crisis tiene entre sus principales causas, según este autor, al movimiento de las matemáticas modernas, y a que tradicionalmente se ha enseñado el producto final de la actividad matemática, es decir, un conjunto de definiciones, axiomas, teoremas y demostraciones que se pretende los estudiantes reproduzcan. Sin embargo, esta visión contradice el enfoque actual, en el que la resolución de problemas es el medio para aprender matemáticas, partiendo de que si el trabajo de los matemáticos es resolver problemas, el aprender matemáticas debiera ser consistente con esto.

En México, la resolución de problemas ha tenido gran importancia en la enseñanza y aprendizaje de las matemáticas en el nivel básico desde los años 90, sin embargo es hasta el 2009 que la resolución de problemas con contextos reales que trasciendan el ámbito escolar es para la Reforma Integral de Educación Media Superior (RIEMS) la finalidad del campo disciplinar de Matemáticas, enfatizando que "El aprendizaje requiere contextualización: los aprendices deben trabajar con tareas auténticas y significativas culturalmente, y necesitan aprender a resolver problemas con sentido" [5].

Tomando en cuenta estos antecedentes, en este diseño didáctico lo que se busca es partir de una situación problema lo suficientemente rica en donde las ideas matemáticas que se pretenden estudiar, en este caso las propiedades de los polígonos, se generen durante el 
proceso de solución, viendo a la resolución de problemas no sólo como un fin del aprendizaje de las matemáticas, sino como el medio ideal para aprenderlas. En el camino, los estudiantes deberán explorar, conjeturar, interpretar y argumentar sus soluciones, impactando así al desarrollo de la comunicación, de resolución de problemas, de construcción e interpretación de modelos matemáticos y de argumentación entre otras competencias, incluidas en el Marco Curricular Común (MCC) de la Educación Media Superior (EMS).

\section{El contexto}

Las investigaciones dentro de la Matemática Educativa arrojan resultados favorables en torno a dos líneas: la resolución de problemas como medio para aprender y el uso de recursos tecnológicos en la enseñanza y aprendizaje de las matemáticas. La propuesta que se presenta incluye ambas. Por un lado se tiene como punto de partida de las actividades didácticas la cuestión problemática del recubrimiento de una superficie plana, y por el otro, además de trabajar con materiales manipulativos y guías de trabajo, se incorpora un software de geometría dinámica, en este caso GeoGebra, como apoyo para la visualización y como herramienta de construcción.

El problema o cuestión es el recubrimiento regular del plano o teselaciones, que se contextualiza en la situación de una compañía que fabrica losetas de concreto en formas poligonales para pisos. Este problema, además de estar relacionado con la creatividad y las manifestaciones artísticas, nos permite partir de un contexto significativo del mundo real que involucra un problema matemático tal y como lo propone Freudenthal [3], el esquema para el estudio de las matemáticas según la Teoría Antropológica de lo Didáctico (TAD) y el enfoque por competencias del MCC.

Algunas actividades de la secuencia que aquí se presenta se hacen en GeoGebra. Este software permite el trazado dinámico de construcciones geométricas, la representación gráfica, el tratamiento algebraico y el cálculo de funciones reales de variable real, sus derivadas e integrales, uniendo geometría, álgebra y cálculo [4]. En GeoGebra los estudiantes pueden probar sus ideas y recibir retroalimentación inmediata permitiendo una exploración "experimental" de las situaciones geométricas y ampliar así su habilidad para descubrir patrones y formular conjeturas, además de promover los procesos de visualización, de construcción y discursivos necesarios para el aprendizaje de la geometría. Además, un aspecto muy importante y que en este proyecto resulta crucial, consiste en que el software es preferentemente utilizado como herramienta de construcción, pues para que los estudiantes construyan objetos matemáticos en ese ambiente, necesitan hacerlo en términos de sus propiedades geométricas: requieren elegir los comandos correctos para lograr los trazos que cumplan con las características de la configuración geométrica buscada.

Con esta secuencia de actividades se estudian los polígonos, tema ubicado en el curso de Matemáticas II, el cual se imparte en segundo semestre de bachillerato general y forma parte del componente de formación básica del currículum. Corresponde al cuarto bloque del curso, el cual lleva por nombre IV: Reconoces las propiedades de los polígonos [6]. 


\section{$3 \quad$ El diseño}

Para sustentar este diseño se tomaron elementos de marcos teóricos de la Matemática Educativa. Por un lado la TAD, creada por el matemático francés Yves Chevallard en los años 90, describe la actividad matemática y el saber que de ella surge en términos de organizaciones o praxeologías matemáticas. Una Organización Matemática (OM) está compuesta por cuatro elementos: tipos de tareas que surgen de una cuestión problemática, tipos de técnicas que permiten resolver los tipos de problemas, tecnologías o discursos que describen y explican las técnicas, y una teoría que fundamenta y organiza dichas tecnologías.

Estudiar matemáticas es la reconstrucción de organizaciones matemáticas, hechas por otros en otro tiempo, bajo otras circunstancias y con otro propósito, y que constituyen el "saber sabio", en la institución en donde se realiza el estudio [1]. La enseñanza consiste en dirigir dicha reconstrucción a partir de una Organización Didática (OD) asociada a la OM por estudiar. La OM, para poder ser reconstruida, sufre una serie de transformaciones, llamada por Chevallard transposición didáctica, bajo una vigilancia epistemológica que debe evitar la ruptura entre el saber "sabio" y el saber a "enseñar".

A partir de lo anterior, antes de realizar el diseño de la secuencia de actividades didácticas, fue necesario definir la cuestión problemática y formular la $\mathrm{OM}$ a reconstruir. Para ello se hizo una clasificación de los tipos de tareas involucrados en el problema del recubrimiento regular del plano (Figura 1) y se decidió, por cuestiones del tiempo con el que se cuenta para el tema en el curso, que solamente se trabajaría con teselaciones con polígonos regulares.

Una vez decidido el tipo de tareas que se abarcaría con el diseño, se continuó con la formulación del conjunto de praxeologías que constituyen la $\mathrm{OM}$ a reconstruir mediante la secuencia de actividades didácticas propuesta. En ella quedaron descritos los tipos de tareas y tareas, las técnicas (estrategias) para resolverlas y las tecnologías (argumentos) que justifican dichas técnicas (Figura 2).

A partir de esta OM se diseñaron las actividades didácticas y sus respectivas hojas de trabajo. Además en algunas de las tareas se trabaja con materiales manipulativos consistentes en figuras de foamy con la forma de los polígonos regulares de 3 a 12 lados, que se utilizan para iniciar la exploración del problema.

Además de la TAD, al tratarse de un tema de estudio de la geometría, se consideró lo expuesto por Raymond Duval acerca del aprendizaje de la geomtetría desde el punto de vista cognitivo, para quien el pensamiento geométrico implica tres tipos de procesos: de visualización, de construcción y de razonamiento [2]. En el diseño se buscó incluir solamente actividades que los desarrollaran, se parte de las construcciones en GeoGebra de las teselaciones que el estudiante debe realizar. Solicitándole en todo momento que describa, explique y argumente sus procedimientos y respuestas, promoviendo de esta manera la competencia comunicativa mediante el apoyo en la visualización.

El problema que se plantea al inicio de la secuencia y que busca brindar un contexto real y cotidiano para los estudiantes es el siguiente:

Recubrir el piso con losetas: La empresa OXICRETO, fabricante de pisos de concreto, 


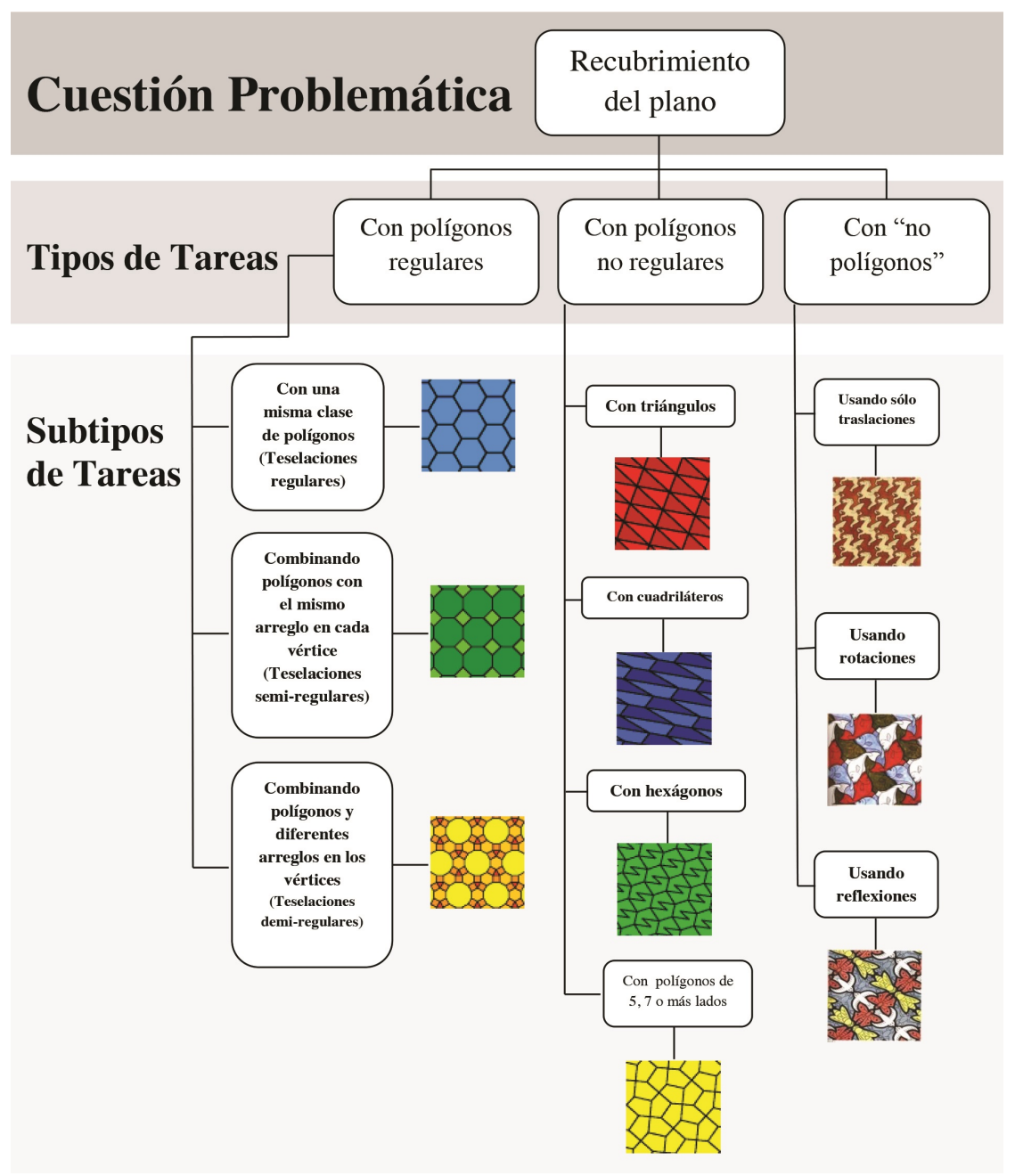

Figura 1: Tipos de tareas

va a lanzar al mercado un nuevo producto: losetas de concreto oxidado con la forma de los polígonos regulares (de 3 a 12 lados). Por esta razón se necesita elaborar un catálogo en el que aparezca la gran variedad de diseños que se pueden hacer combinando las losetas. Además, para cada diseño, se debe hacer la estimación de las losetas que se necesitan de cada figura para cubrir un metro cuadrado. Todas las losetas tienen 20 centímetros de lado.

\section{Puesta en escena}

Se realizaron dos puestas en escena, la primera de carácter experimental y la segunda en el curso normal de geometría para la que fue elaborada, con la finalidad de probar la validez experimental de la propuesta aunque sin el rigor metodológico que exigiría un trabajo de investigación.

A partir de la primera aplicación se hicieron ajustes al diseño, se corrigieron los errores 


\begin{tabular}{|c|c|c|c|}
\hline $\begin{array}{l}\text { Subtipos } \\
\text { de Tarea }\end{array}$ & Tareas & Técnicas & Tecnologías \\
\hline \multirow{7}{*}{$\begin{array}{c}\text { Recubrir el plano } \\
\text { con una misma clase } \\
\text { de polígonos } \\
\text { regulares } \\
\text { (Teselaciones } \\
\text { regulares) } \\
\mathbf{T}_{\mathbf{r}}\end{array}$} & $\begin{array}{l}\text { Tr1 Recubre la superficie señalada } \\
\text { con las piezas de foamy disponibles } \\
\text { sin dejar huecos y sin traslapos. }\end{array}$ & $\begin{array}{l}\tau_{\mathbf{r} 1 \text { Exploración con las piezas de }} \\
\text { foamy con la forma de los polígonos } \\
\text { regulares. }\end{array}$ & \multirow{2}{*}{$\begin{array}{l}\theta_{\text {r1 }} \text { Polígonos regulares: } \\
\text { Propiedades de los ángulos }\end{array}$} \\
\hline & $\begin{array}{l}\text { Tr2 Dibuja con GeoGebra las } \\
\text { teselaciones regulares ya hechas en } \\
\text { foamy. }\end{array}$ & $\begin{array}{l}\tau_{\mathbf{r} 2} \text { Dibujo usando "copiar" y } \\
\text { "pegar". }\end{array}$ & \\
\hline & \multirow[b]{2}{*}{$\begin{array}{l}\text { Tr3 Construye con GeoGebra las } \\
\text { teselaciones regulares. }\end{array}$} & $\begin{array}{l}\tau_{\text {r3a Construcción usando los }} \\
\text { comandos traslación y rotación. }\end{array}$ & \multirow[b]{2}{*}{$\begin{array}{c}\theta_{\text {r3 } \text { Isometrías: }} \\
\text { Traslación y rotación } \\
\text { Definición de teselación regular } \\
\text { Distinción entre dibujo y } \\
\text { construcción }\end{array}$} \\
\hline & & $\begin{array}{l}\tau_{\mathbf{r} 3 \mathbf{b}} \text { Construcción de una figura } \\
\text { mínima. } \\
\text { Construcción a partir de una figura } \\
\text { mínima trasladándola en dos } \\
\text { direcciones diferentes. }\end{array}$ & \\
\hline & $\begin{array}{l}T_{\text {r4 }} \text { Construye con GeoGebra las } \\
\text { teselaciones duales de las regulares. }\end{array}$ & $\begin{array}{l}\tau_{\mathbf{r} 4} \text { Construcción de las teselaciones } \\
\text { duales de las regulares. }\end{array}$ & $\begin{array}{c}\theta_{\mathbf{r} 4} \text { Definición de teselaciones } \\
\text { duales }\end{array}$ \\
\hline & \multirow[t]{2}{*}{$\begin{array}{l}\text { Tr5 Responde: ¿Por qué los panales } \\
\text { de las abejas son teselaciones } \\
\text { hexagonales? }\end{array}$} & $\begin{array}{l}\tau_{\mathbf{r} 5 \mathbf{a} \text { Exploración con applet para }} \\
\text { comparar geométricamente y } \\
\text { contrastar numéricamente las áreas } \\
\text { de un triángulo, un cuadrado y un } \\
\text { hexágono con el mismo perímetro. }\end{array}$ & \multirow[t]{2}{*}{$\begin{array}{l}\theta_{\mathrm{r} 5} \text { Perímetro y área de los } \\
\text { polígonos regulares }\end{array}$} \\
\hline & & $\begin{array}{l}\tau_{\mathbf{r} 5 \mathbf{b}} \text { Cálculo analítico de las áreas } \\
\text { de los } 3 \text { polígonos regulares }(3,4 \text { y } 6 \\
\text { lados) para generalizar lo observado. }\end{array}$ & \\
\hline \multirow{2}{*}{$\begin{array}{c}\text { Recubrir el plano } \\
\text { combinando } \\
\text { polígonos regulares } \\
\text { con el mismo arreglo } \\
\text { en cada vértice } \\
\text { (Teselaciones semi- } \\
\text { regulares) } \\
\mathbf{T}_{\mathbf{s}}\end{array}$} & $\begin{array}{l}\text { Ts1 Recubre la superficie señalada } \\
\text { con las piezas de foamy disponibles } \\
\text { sin dejar huecos y sin traslapos. }\end{array}$ & $\begin{array}{l}\tau_{\text {s1 Exploración con las piezas de }} \\
\text { foamy con la forma de los polígonos } \\
\text { regulares. }\end{array}$ & $\begin{array}{l}\theta_{\mathrm{s} 1} \text { Polígonos regulares: } \\
\text { Propiedades de los ángulos }\end{array}$ \\
\hline & $\begin{array}{l}\mathrm{T}_{\mathbf{s} 2} \text { Construye con GeoGebra las } \\
\text { teselaciones semi-regulares. }\end{array}$ & $\begin{array}{l}\tau_{\mathbf{s} 2} \text { Construcción de una figura } \\
\text { mínima. } \\
\text { Construcción a partir de una figura } \\
\text { mínima trasladándola en dos } \\
\text { direcciones diferentes. }\end{array}$ & $\begin{array}{c}\theta_{\text {s2 Isometrías: }} \\
\text { Traslación y rotación } \\
\text { Definición de teselación semi- } \\
\text { regular }\end{array}$ \\
\hline \multirow{2}{*}{$\begin{array}{c}\text { Recubrir el plano } \\
\text { combinando } \\
\text { polígonos regulares } \\
\text { y diferentes arreglos } \\
\text { en los vértices } \\
\text { (Teselaciones demi- } \\
\text { regulares) } \\
\mathbf{T}_{\mathbf{d}}\end{array}$} & $\begin{array}{l}\text { Td1 Recubre la superficie señalada } \\
\text { con las piezas de foamy disponibles } \\
\text { sin dejar huecos y sin traslapos. }\end{array}$ & $\begin{array}{l}\tau_{\mathbf{d} 1 \text { Exploración con las piezas de }} \\
\text { foamy con la forma de los polígonos } \\
\text { regulares. }\end{array}$ & $\begin{array}{l}\theta \text { d1 Polígonos regulares: } \\
\text { Propiedades de los ángulos }\end{array}$ \\
\hline & $\begin{array}{l}\text { Td2 Construye con GeoGebra las } \\
\text { teselaciones demi-regulares. }\end{array}$ & $\begin{array}{l}\tau_{\mathrm{d} 2} \text { Construcción de una figura } \\
\text { mínima. } \\
\text { Construcción a partir de una figura } \\
\text { mínima trasladándola en dos } \\
\text { direcciones diferentes. }\end{array}$ & $\begin{array}{c}\theta_{\mathbf{d} 2} \text { Isometrías: } \\
\text { Traslación y rotación } \\
\text { Definición de teselación demi- } \\
\text { regular }\end{array}$ \\
\hline
\end{tabular}

Figura 2: Organización Matemática

detectados y se modificó la redacción de algunas preguntas para facilitar su comprensión. Se incluyeron aspectos que no se habían contemplado en la primera versión, como resaltar que hay arreglos de polígonos que suman $360^{\circ}$ y no forman una teselación lo que da pie a introducir una expresión importante en matemáticas: la "condición necesaria pero no suficiente". También se añadió un nuevo applet que brinda dinamismo a la tabla impresa en las hojas de trabajo anteriores, en la que se proponía explorar las características de interés en los polígonos regulares hasta el dodecágono. Este cambio, que da dinamismo a la construcción de la tabla que proporciona el applet incorporado en esta segunda versión, permite extender la exploración a un polígono de $n$ lados, incluyendo la suma de sus ángulos interiores, el valor de su ángulo interior, el número de polígonos de cada tipo que se necesitan para cubrir $360^{\circ}$ y la representación geométrica del polígono. Además se incorporó un cuestionario al término de la secuencia de actividades para tener información sobre el nivel de logro de los desempeños que se esperan de los estudiantes al concluir el Bloque IV según el programa de Matemáticas II. 


\section{Conclusiones}

En las puestas en escena se obtuvieron buenos resultados, ya que no sólo se cubrieron los propósitos del tema de polígonos, sino que además, al trabajar con teselaciones, se utilizaron transformaciones isométricas como la traslación y la rotación, se practicaron descripciones, explicaciones y argumentaciones (procesos discursivos) y se realizaron construcciones con GeoGebra, impactando todo ello en un mayor desarrollo del pensamiento geométrico de los estudiantes y en la movilización de competencias, tanto genéricas como disciplinares. Algunos de los diseños de configuraciones para el catálogo de la empresa de pisos hechos por los estudiantes se muestran en la Figura 3.
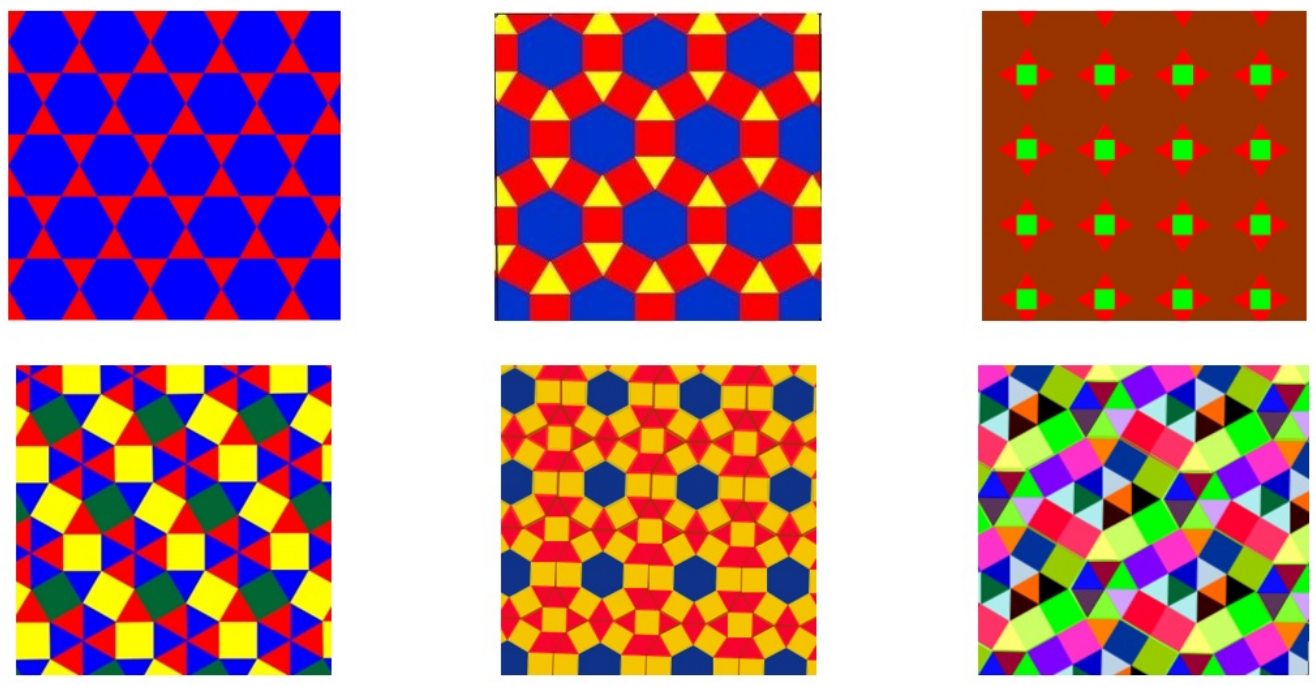

Figura 3: Algunos diseños del catálogo hechos por los estudiantes

Fue muy importante la utilización de las teselaciones como contexto para el estudio de los polígonos. Además de ser atractivo y cercano para los estudiantes, y de estar vinculado con la creatividad y la sensibilidad a las manifestaciones artísticas, permitió que el problema del que surgen, es decir, el recubrimiento del plano, le diera sentido al estudio de los polígonos.

Se considera que esta propuesta para el estudio de los polígonos, aunque logró integrar la OM y la OD en puestas en escena que se pueden calificar como exitosas, no constituye una organización praxeológica absoluta. Su riqueza didáctica la constituye precisamente la posibilidad de ser, más que replicada, transpuesta, es decir, reconstruida a partir de las condiciones particulares del grupo y del profesor. En la medida que las modificaciones favorezcan de mejor manera la movilización de los procesos cognitivos de los estudiantes, esta propuesta habrá de satisfacer su razón de ser.

\section{Referencias}

[1] Chevallard, Y. El análisis de las prácticas docentes en la teoría antropológica de lo didáctico. (1999), Recuperado el 6 de abril del 2013 de la página 
http://servidor-opsu.tach.ula.ve/profeso/guerr_o/praticamatema /referencias/practica_marcosteoricos3/Chevallard_Teoria_Antropologica.pdf

[2] Duval, R. Geometry from a cognitive point of view. En C., Mammana \& V., Villani (Eds.), Perspective the Teaching of de Geometry for the 21st Century (pp. 37-51). (1998), Dordrecht, Netherlands: Kluwer Academir in Mathematics.

[3] Freundenthal, H. Problemas Mayores de la Educación Matemática (pp. 133-159). (1981), Berkeley: Educational Studies in Mathematics.

[4] García, M. Evolución de actitudes y competencias matemáticas en estudiantes de secundaria al introducir GeoGebra en el aula. (2011), Tesis doctoral no publicada. Universidad de Almería. España. Recuperado en mayo del 2013 de la página www.geogebra.org/en/upload/files/Tesis_MariadelMarGarciaLopez.pdf

[5] Secretaría de Educación Pública. Lineamientos para la práctica educativa a partir del enfoque educativo basado en competencias. (Coloquio de experiencias docentes). (2010), Dirección General de Bachillerato. Recuperado en junio del 2013 de la página http://www.dgb.sep.gob.mx/02-m1/03-iacademica/00-otros/Linpractica_educa.pdf

[6] Secretaría de Educación Pública. Matemáticas II. Serie Programas de Estudio. (2013), Dirección General de Bachillerato. Recuperado en junio del 2013 de la página http://www.dgb.sep.gob.mx/02-m1/03-iacademica/01programasdeestudio/cfb_2sem/MATEMATICAS-II.pdf

[7] Villani, V. Perspectivas en la Enseñanza de la Geometría para el Siglo XXI. (1998), Kluwer Academic Publishers. Canadá. 